\title{
"A multiplexed recombinase polymerase amplification assay to detect intestinal protozoa"
}

\section{Zachary Crannell, Alejandro Castellanos-Gonzales, Gayatri Nair, Rojelio Mejia, A. Clinton White, Rebecca Richards-Kortum*}

Zachary Crannell 1980 W Getaway Trail

Flagstaff, AZ 86005

Alejandro Castellanos-Gonzales

UTMB Department of Internal Medicine

Division of Infectious Diseases

301 University Blvd

Galveston, TX 77555.0435

Gayatri Nair

UTMB Department of Internal Medicine

Division of Infectious Diseases

1709 Dryden Rd \# 1700

Houston, TX 77030

Rojelio Mejia

Department of Pediatrics, Section of Tropical Medicine; Department of Medicine, Section of Infectious Diseases; National School of Tropical Medicine

Baylor College of Medicine

1709 Dryden Rd \# 1700

Houston, TX 77030

A. Clinton White

UTMB Department of Internal Medicine

Division of Infectious Diseases

301 University Blvd, Mary Moody Northen, Rm 4.230

Galveston, Texas 77555-0435

*Rebecca Richards-Kortum

Malcolm Gillis University of Professor, Professor of Bioengineering and ECE

Department of Bioengineering

Rice University

Houston, TX 77030

Fax: (713) 348-5877

E-mail: rkortum@rice.edu 
Supplemental Table 1: Double-stranded DNA standards used for bench-top characterization.

\begin{tabular}{|c|c|c|}
\hline Target & Accession No. & DNA sequence \\
\hline Giardia lamblia & X85958.1 & $\begin{array}{l}\text { 5'-TAC GCT CAC CCA GAC GAT GGA CAA GCC CGA CGA } \\
\text { CCT CAC CCG CAG TGC GAC CGA GAC GGC GGT CAA GCT } \\
\text { CAG CAA CAT GAA CCA GCG CGT CAG CAG GTT CCA CGA } \\
\text { CAA GAT GGA GAA CGA GAT CGA GGT CCG CCG CGT } \\
\text { CGA CGA CGA CAC GCG CGT GAA GAT GAT CAA GGA } \\
\text { CGC CAT CGC ACA-3' }\end{array}$ \\
\hline $\begin{array}{l}\text { Entamoeba } \\
\text { histolytica }\end{array}$ & AB608092.1 & $\begin{array}{l}\text { 5'-GTA CAA AAT GGC CAA TTC ATT CAA TGA ATT GAG } \\
\text { AAA TGA CAT TCT AAG TGA GTT AGG ATG CCA CGA CAA } \\
\text { TTG TAG AAC ACA CAG TGT TTA ACA AGT AAC CAA TGA } \\
\text { GAA TTT CTG ATC TAT CAA TCA GTT GGT AGT-3' }\end{array}$ \\
\hline $\begin{array}{l}\text { Cryptosporidium } \\
\text { species }\end{array}$ & AF115377.1 & $\begin{array}{l}\text { 5'-GTG GCA ATG ACG GGT AAC GGG GA ATT AGG GTT } \\
\text { CGA TTC CGG AGA GGG AGC CTG AGA AAC GGC TAC CAC } \\
\text { ATC TAA GGA AGG CAG CAG GCG CGC AAA TTA CCC AAT } \\
\text { CCT AAT ACA GGG AGG TAG TGA CAA GAA ATA ACA ATA } \\
\text { CAG GAC TTT TTG GTT TTG TAA TTG GAA TGA GTT AAG } \\
\text { TAT AAA CCC CTT TAC AAG TAT CAA TT-3' }\end{array}$ \\
\hline
\end{tabular}

